\title{
CLASSIFICATION THEOREM FOR MENGER MANIFOLDS
}

\author{
A. CHIGOGIDZE
}

(Communicated by James E. West)

\begin{abstract}
We introduce the notion of the $n$-homotopy kernel of a Menger manifold and prove the following theorem: Menger manifolds are $n$-homotopy equivalent if and only if the $n$-homotopy kernels are homeomorphic.
\end{abstract}

This paper is devoted to some problems induced by the internal development of Menger manifold theory. It is closely related to previous papers [1, 8, 3-5] and can be considered as their natural continuation. We gave a detailed discussion of certain phenomena in [5] that are inherent in this theory (in comparison with Hilbert cube manifold theory) and that are clearly manifested even in the formulations of such important components of the theory as stability and triangulation theorems $[8,5]$ as well as the open embedding theorem [5]. Let us consider the last proposition more formally (see $\S 1$ for definitions). It states that each Menger $(n+1)$-manifold $N$ contains a $Z$-set $Z$ satisfying the following conditions: (1) $Z$ is homeomorphic to $N$; (2) the complement $N-Z$ admits an open embedding into $M^{n+1}$ (throughout the paper $M^{k}$ denotes the $k$-dimensional universal Menger compactum). It should be observed (it was remarked in [5], too) that there is a certain arbitrariness in the above formulation connected with the choice of homeomorphism from condition (1). It will be shown that under minimal restrictions the mentioned arbitrariness can be eliminated. At the same time this specifies the second condition, because in this way the complement $N-Z$ will acquire completely correct sense and will depend only on the Menger $(n+1)$-manifold $N$. Moreover, this complement (which will be called an $n$-homotopy kernel of $N$ and will be denoted by $\operatorname{Ker}(N))$ becomes a very important object associated with $N$. Indeed, if we bear in mind the strong parallel between Hilbert cube manifold theory and Menger $(n+1)$ manifold theory then we shall be able to conclude confidently that $\operatorname{Ker}(N)$ plays the role of the product $N \times[0,1)$ (compare our modified open embedding theorem below and Chapman's open embedding theorem for Hilbert cube manifolds [2]). Moreover, the consideration of $n$-homotopy kernels gives us the possibility to solve the problem of classification of Menger $(n+1)$-manifolds by $n$-homotopy type. Our main result states in this connection that Menger $(n+1)$-manifolds are $n$-homotopy equivalent if and only if their $n$-homotopy kernels are homeomorphic (compare with Chapman's theorem that states that

Received by the editors September 14, 1990.

1991 Mathematics Subject Classification. Primary 55P15; Secondary 54F35. 
two Hilbert cube manifolds $X$ and $Y$ are homotopy equivalent if and only if the products $X \times[0,1)$ and $Y \times[0,1)$ are homeomorphic [2]). Of course, there is nothing to do in the compact case, because, by Bestvina's theorem [1], $n$-homotopy equivalent compact Menger $(n+1)$-manifolds are homeomorphic.

This paper is organized as follows. In $\S 1$ we present the definitions and results we need from the theory of Menger manifolds. In $\S 2$ we introduce the concept of $n$-homotopy kernel and prove the main result.

\section{Preliminary facts}

All the spaces in this paper are assumed to be locally compact and metrizable and maps are assumed to be continuous. $C(X, Y)$ denotes the set of all continuous maps from $X$ to $Y$, endowed with the limitation topology (uniform convergence in all equivalent metrics). Let us recall that if $X$ is compact then this topology coincides with the compact-open topology.

A closed subset $Z$ of $X$ is called a $Z$-set (in $X$ ) if the identity map $\operatorname{id}_{X}$ can be approximated arbitrarily closely (in the topology indicated above) by maps whose ranges do not intersect $Z$. An embedding whose range is a $Z$-set is called a $Z$-embedding.

The $k$-dimensional universal Menger compactum $M^{k}$ is understood to be the compactum obtained from the $(2 k+1)$-dimensional unit cube by the standard Cantor process of "deleting interiors of central cubes" $[9,1]$. A $k$-dimensional Menger manifold (briefly, $M^{k}$-manifold) is defined to be a space in which each point has an open neighborhood homeomorphic to an open subspace of $M^{k}$. The following remarkable result, which gives a topological characterization of Menger manifolds, is due to Bestvina [1].

Theorem 1.1. Let $X$ be an $(n+1)$-dimensional locally compact LC $C^{n}$-space. Then the following conditions are equivalent:

(i) $X$ is a Menger $(n+1)$-manifold;

(ii) if $B_{0}$ is a closed subset of a locally compact space $B$ with $\operatorname{dim} B \leq$ $n+1$ then any proper map $f: B \rightarrow X$, such that the restriction $f \mid B_{0}$ is a $Z$-embedding, can be approximated (up to a given open cover of $X$ ) by a $Z$ embedding that agrees with $f$ on $B_{0}$;

(iii) $X$ satisfies the disjoint $(n+1)$-disks property, i.e., any map of the $(n+1)$ dimensional cube into $X$ can be approximated arbitrarily closely by maps having disjoint images.

As was shown in [1] an adequate homotopy language for Menger $(n+1)$ manifold theory is based on the concept of (proper) $M^{n+1}$-homotopy between maps. Its definition is cumbersome and, moreover, assumes a fairly high degree of local connectedness of the range. This involves certain inconveniences. In [3, 4] we gave another, much simpler, and more convenient variant of this relation not assuming any restrictions on the range. It was shown in [3] that these two notions coincide for maps between at most $(n+1)$-dimensional locally compact $L C^{n}$-spaces, and so below we shall use only our definition and respectively the corresponding results from [1] will be translated having in mind this agreement. The definition is as follows.

Definition 1.1. Two (proper) maps $f, g: X \rightarrow Y$ are said to be (properly) $n$-homotopic (written $f \simeq^{n} g$ and $f \simeq_{p}^{n} g$, respectively) if the compositions 
$f \cdot h$ and $g \cdot h$ are (properly) homotopic in the usual sense for any (proper) map $h: Z \rightarrow X$ of any at most $n$-dimensional space $Z$ into $X$.

The concept of (proper) $n$-homotopy equivalence is defined in an obvious way.

It should be observed especially that the relations of such type were considered by Fox [10] and Whitehead [12]. The following two propositions directly follow from our definition and corresponding results of Bestvina [1].

Theorem 1.2. Let $Z_{1}$ and $Z_{2}$ be $Z$-sets in a Menger $(n+1)$-manifold $N$ and let $h: Z_{1} \rightarrow Z_{2}$ be a homeomorphism. Denote by $i_{j}$ the inclusion map $Z_{j} \hookrightarrow N$ $(j=1,2)$. If $i_{1} \simeq_{p}^{n} i_{2} \cdot h$ then $h$ extends to a homeomorphism $H: N \rightarrow N$.

Theorem 1.3. Properly $n$-homotopy equivalent Menger $(n+1)$-manifolds are homeomorphic.

The following proposition [1] will be needed in $\S 2$.

Theorem 1.4. Each proper $U V^{n}$-surjection between Menger $(n+1)$-manifolds can be approximated arbitrarily closely by homeomorphisms.

Before formulating the following two resolution theorems, let us recall that a map $f: X \rightarrow Y$ is said to be $k$-invertible if for any map $g: Z \rightarrow Y$ with $\operatorname{dim} Z \leq k$ there exists a map $h: Z \rightarrow X$ such that $f \cdot h=g$. The following result is due to Dranishnikov [8]. Here, $Q$ denotes the Hilbert cube.

Theorem 1.5. There exists an $(n+1)$-invertible $U V^{n}$-surjection $f_{n}: M^{n+1} \rightarrow Q$ satisfying the following condition:

(i) if $X$ is a locally compact $L C^{n}$-space lying in $Q$, then its inverse image $f_{n}^{-1}(X)$ is a Menger $(n+1)$-manifold.

Of course, Dranishnikov's map has some other important properties as well. But we shall not use them in this paper. Another similar result is the following resolution theorem.

Theorem 1.6. For each locally compact polyhedron $K$ there exists an $(n+1)$ invertible proper $U V^{n}$-surjection $f_{K}^{n}: M_{K}^{n+1} \rightarrow K$ of some Menger $(n+1$ manifold $M_{K}^{n+1}$ onto $K$ satisfying the following conditions:

(i) if $L$ is a closed subpolyhedron of $K$ then its inverse image $\left(f_{K}^{n}\right)^{-1}(L)$ is a Menger $(n+1)$-manifold;

(ii) if $L$ is a closed subpolyhedron of $K$ and $Z$ is a $Z$-set in $L$, then the inverse image $\left(f_{K}^{n}\right)^{-1}(Z)$ is a $Z$-set in $\left(f_{K}^{n}\right)^{-1}(L)$.

It should be observed that the construction of the map $f_{K}^{n}$ was given in fact in [6], but I could not see at that time that conditions (i) and (ii) were satisfied. After that, some concrete considerations showed that it became very important to have a resolution map satisfying property (ii) (see [5, problem 2]) and, moreover, as we shall see below this property is a main technical moment in proving our classification theorem. A complete proof of Theorem 1.6 was given very recently in [7].

The next proposition that will be needed in the sequel very often is an easy consequence of the well-known properties of proper $U V^{n}$-maps between locally compact $L C^{n}$-spaces $[11,1]$ and of Theorem 1.3. 
Proposition 1.1. Two Menger $(n+1)$-manifolds admitting proper $U V^{n}$-surjections onto the same $L C^{n}$-space are homeomorphic.

The following two propositions will be useful also. Their proofs were given in [5] and [1], respectively.

Proposition 1.2. Each Menger $(n+1)$-manifold admits an $(n+1)$-invertible proper $U V^{n}$-surjection onto some Hilbert cube manifold.

Proposition 1.3. Let $f: M \rightarrow X$ be a proper $U V^{n}$-surjection of a Menger $(n+1)$ manifold and let $Z$ be a $Z$-set in $M$. If $g: Z \rightarrow X$ is any proper map properly $n$-homotopic to $f \mid Z$, then there exists a proper $U V^{n}$-surjection $h: M \rightarrow X$ such that $h \mid Z=g$.

\section{THE $n$-HOMOTOPY KERNEL AND THE CLASSIFICATION THEOREM}

Consider a Menger $(n+1)$-manifold $M$ and two $Z$-embeddings $f, g: M \rightarrow$ $M$ each of which is properly $n$-homotopic to the identity map $\operatorname{id}_{M}$. Then the homeomorphism $g \cdot f^{-1}: f(M) \rightarrow g(M)$ is properly $n$-homotopic to $\operatorname{id}_{f(M)}$ and, consequently, by Theorem 1.2, there exists a homeomorphism $h: M \rightarrow M$ extending $g \cdot f^{-1}$. Then the restriction $h \mid(M-f(M))$ is a homeomorphism between the complements $M-f(M)$ and $M-g(M)$. This shows that the following definition is correct and does not depend on the choice of the corresponding $Z$-embedding.

Definition 2.1. An $n$-homotopy kernel $\operatorname{Ker}(M)$ of a Menger $(n+1)$-manifold $M$ is defined to be the complement $M-f(M)$ where $f: M \rightarrow M$ is an arbitrary $Z$-embedding properly $n$-homotopic to $\operatorname{id}_{M}$.

Proposition 2.1. If a Menger $(n+1)$-manifold $N$ admits a proper $U V^{n}$-surjection onto the product $M \times[0,1)$, where $M$ is a Menger $(n+1)$-manifold, then $N$ is homeomorphic to $\operatorname{Ker}(M)$.

Proof. By Proposition 1.1, it suffices to show that $\operatorname{Ker}(M)$ admits a proper $U V^{n}$-surjection onto the product $M \times[0,1)$. By Theorem 1.5 , we can fix a proper $U V^{n}$-surjection $f: M_{1} \rightarrow M \times[0,1]$ where $M_{1}$ is also a Menger $(n+1)$ manifold. Consider the quotient space $M_{2}$ of $M_{1}$ with respect to the partition whose nontrivial elements are fibers $f^{-1}(m, 1)$ over the $Z$-set $M \times\{1\}$ of the product $M \times[0,1]$. Clearly, by Theorem $1.1, M_{2}$ is a Menger $(n+$ 1)-manifold. Moreover, if we consider the naturally induced $U V^{n}$-surjection $g: M_{2} \rightarrow M \times[0,1]$, then we conclude, by Proposition 1.1 , that $M_{1}$ and $M_{2}$ are even homeomorphic. Now it is easy to see that the set $g^{-1}(M \times\{1\})$ is a $Z$-set in $M_{2}$ and the restriction $g \mid g^{-1}(M \times\{1\})$ is a homeomorphism. By Theorem 1.4, the composition $\pi_{M} g$, where $\pi_{M}: M \times[0,1] \rightarrow M$ is the natural projection, can be arbitrarily closely approximated by homeomorphisms. Particularly, there exists a homeomorphism $h: M_{2} \rightarrow M$ that is properly $n$ homotopic to $\pi_{M} g$. Then the map $r=h \cdot g^{-1} \cdot i$, where $i: M \rightarrow M \times$ $\{1\}$ is a natural homeomorphism, is a $Z$-embedding properly $n$-homotopic to $\operatorname{id}_{M}$. Indeed, $r=h \cdot g^{-1} \cdot i \simeq_{p}^{n} \pi_{M} \cdot g \cdot g^{-1} \cdot i=\pi_{M} \cdot i=\mathrm{id}_{M}$. Thus, by Definition 2.1, we conclude that the complement $M-r(M)$ is homeomorphic to $\operatorname{Ker}(M)$. Consequently the space $M_{2}-g^{-1}(M \times\{1\})=h^{-1}(M-r(M))$ is also homeomorphic to $\operatorname{Ker}(M)$. It only remains to note that the space $M_{2}$ $g^{-1}(M \times\{1\})$ admits a proper $U V^{n}$-surjection onto the product $M \times[0,1)$. 
Comparing the above argument with the proof of an open embedding theorem for Menger $(n+1)$-manifolds [5, Theorem 11], we immediately obtain the following modified version.

Theorem 2.1. The n-homotopy kernel of each Menger $(n+1)$-manifold admits an open embedding into $M^{n+1}$.

One can easily verify that the Menger compactum $M^{n+1}$ without a point is homeomorphic to its $n$-homotopy kernel. The following proposition gives a whole class of "stable" Menger $(n+1)$-manifolds (i.e., Menger $(n+1)$-manifolds that are homeomorphic to their $n$-homotopy kernels).

Proposition 2.2. For each Menger $(n+1)$-manifold $M$, the spaces $\operatorname{Ker}(M)$ and $\operatorname{Ker}(\operatorname{Ker}(M))$ are homeomorphic.

Proof. By Proposition 1.2, we can fix a proper $U V^{n}$-surjection $g: M \rightarrow X$ of $M$ onto some Hilbert cube manifold $X$. By Theorem 1.5 and Proposition 2.1, there exists a proper $U V^{n}$-surjection $f: \operatorname{Ker}(M) \rightarrow M \times[0,1)$. The same reasons show that there exists a proper $U V^{n}$-surjection $h: \operatorname{Ker}(\operatorname{Ker}(M)) \rightarrow$ $\operatorname{Ker}(M) \times[0,1)$. Consequently we have two proper $U V^{n}$-surjections:

$$
p=(g \times \mathrm{id}) \cdot f: \operatorname{Ker}(M) \rightarrow X \times[0,1)
$$

and

$$
q=(p \times \mathrm{id}) \cdot h: \operatorname{Ker}(\operatorname{Ker}(M)) \rightarrow X \times[0,1) \times[0,1),
$$

where id denotes the identity map of $[0,1)$. Since $X$ is a Hilbert cube manifold, the product $X \times[0,1]$ is homeomorphic to $X$ [2]. Noting that the spaces $[0,1) \times[0,1)$ and $[0,1] \times[0,1)$ are homeomorphic, we have $X \times[0,1) \times[0,1) \approx X \times[0,1] \times[0,1) \approx X \times[0,1)$. Consequently, Menger $(n+1)$-manifolds $\operatorname{Ker}(\operatorname{Ker}(M))$ and $\operatorname{Ker}(M)$ admit proper $U V^{n}$-surjections onto the same Hilbert cube manifold. It remains to use Proposition 1.1.

Proposition 2.3. Let $M$ be a Menger $(n+1)$-manifold. If $A$ is a $Z$-set in $\operatorname{Ker}(M)$, then the spaces $\operatorname{Ker}(M)$ and $\operatorname{Ker}(\operatorname{Ker}(M)-A$ ) are homeomorphic.

Proof. As in the proof of the preceding proposition we can fix three proper $U V^{n}$-surjections $g: M \rightarrow X, f: \operatorname{Ker}(M) \rightarrow M \times[0,1)$, and $p=(g \times$ id $)$. $f: \operatorname{Ker}(M) \rightarrow X \times[0,1)$, where $X$ is some Hilbert cube manifold and id denotes the identity map of $[0,1)$. Now we redefine the map $p$ in such a way that the set $p(A)$ will be a $Z$-set in $X \times[0,1)$. For this consider any $Z$ embedding $r: A \rightarrow X \times[0,1)$ properly $n$-homotopic to the restriction $p \mid A$. By Proposition 1.3, there exists a proper $U V^{n}$-surjection $q: \operatorname{Ker}(M) \rightarrow X \times[0,1)$ such that $q \mid A=r$. Moreover, as in the beginning of the proof of Proposition 2.1 , we can additionally suppose that $A=q^{-1} q(A)$. Consequently the restriction $q \mid(\operatorname{Ker}(M)-A): \operatorname{Ker}(M)-A \rightarrow X \times[0,1)-q(A)$ is a proper $U V^{n}$-surjection. As above this implies (together with Theorem 1.5 and Proposition 2.1) that $\operatorname{Ker}(\operatorname{Ker}(M)-A)$ admits a proper $U V^{n}$-surjection onto the product $(X \times[0,1)-q(A)) \times[0,1)$. By the same reasons, there exists a proper $U V^{n}$-surjection of $\operatorname{Ker}(\operatorname{Ker}(M))$ onto the product $X \times[0,1) \times[0,1)$. By Proposition 2.2, $\operatorname{Ker}(M)$ and $\operatorname{Ker}(\operatorname{Ker}(M))$ are homeomorphic. Thus, by Proposition 1.1, it suffices to show that the spaces $(X \times[0,1)-q(A)) \times[0,1)$ and $X \times[0,1) \times[0,1)$ are homeomorphic. Indeed, since $q(A)$ is a $Z$-set in $X \times[0,1)$, we conclude that the Hilbert cube manifolds $X \times[0,1)$ and 
$X \times[0,1)-q(A)$ are homotopy equivalent. Then, by Chapman's theorem [2], the products $X \times[0,1) \times[0,1)$ and $(X \times[0,1)-q(A)) \times[0,1)$ are homeomorphic.

Proposition 2.4. Let a Menger $(n+1)$-manifold $M$ be a $Z$-set of a Menger $(n+1)$-manifold $N$ and the inclusion $i: M \hookrightarrow N$ be an $n$-homotopy equivalence. Then there exists a Z-set $A$ in $N$ such that the complement $N-A$ is homeomorphic to $\operatorname{Ker}(M)$.

Proof. By the triangulation theorem for Menger $(n+1)$-manifolds [5], there exist $(n+1)$-invertible proper $U V^{n}$-surjections $\varphi: M \rightarrow L$ and $\psi: N \rightarrow K_{1}$, where $L$ and $K_{1}$ are $(n+1)$-dimensional locally compact polyhedra. $(n+1)$ invertibility of $\varphi$ implies the existence of a proper map $s: L \rightarrow M$ with $\varphi \cdot s=$ $\mathrm{id}_{L}$. Then the composition $\psi \cdot i \cdot s: L \rightarrow K_{1}$ is properly homotopic to some proper PL-map $p: L \rightarrow K_{1}$. Form the mapping cylinder, $M(p)=K$ of the map $p$. Recall that it is the space formed from the disjoint union $(L \times[0,1]) \oplus K_{1}$, by identifying $(l, 1)$ with $p(l), l \in L$. At the same time we identify $L$ with $L \times\{0\}$. Clearly $L \times[0,1)$ can be considered as an open subspace of $K$. Since $p$ is a proper PL-map, $L \times\{0\}$ and $K_{1}$ are subpolyhedra of the polyhedron $K$. Let $c: K \rightarrow K_{1}$ be the collapse to the base, i.e., the natural retraction defined by sending $(l, t)$ to $p(l)$. Clearly $c$ is a proper $U V^{n}$-surjection that is homotopy equivalent.

Now we fix an $(n+1)$-invertible proper $U V^{n}$-surjection $f_{K}^{n}: M_{K}^{n+1} \rightarrow K$ satisfying the conditions of Theorem 1.6. Clearly the composition $c \cdot f_{K}^{n}: M_{K}^{n+1} \rightarrow K_{1}$ is a proper $U V^{n}$-surjection and hence, by Proposition 1.1, the Menger $(n+1)$ manifolds $M_{K}^{n+1}$ and $N$ are homeomorphic. By Theorem 1.6, the inverse image $\left(f_{K}^{n}\right)^{-1}(L \times\{0\})$ is a Menger $(n+1)$-manifold that, again by Proposition 1.1 , is homeomorphic to $M$. One can easily verify, using our assumption and the specifics of the above construction, that the natural inclusion of $\left(f_{K}^{n}\right)^{-1}(L \times\{0\})$ into $M_{K}^{n+1}$ is an $n$-homotopy equivalence. Moreover, by Theorem 1.6, the above inverse image is a $Z$-set in $M_{K}^{n+1}$ (since $L \times\{0\}$ is a $Z$-set in $K$ ). Now redenoting the above objects for simplicity, we shall have the following situation: a proper $U V^{n}$-surjection $f: N \rightarrow K$ satisfying the conditions of Theorem 1.6 is given, $M=f^{-1}(L \times\{0\})$ is a $Z$-set in $N$, and the inclusion $M \hookrightarrow N$ is an $n$-homotopy equivalence. Clearly $K-K_{1}=L \times[0,1)$ and hence its inverse image $f^{-1}(L \times[0,1))$ admits a proper $U V^{n}$-surjection onto $L \times[0,1)$. On the other hand, $\operatorname{Ker}(M)$ admits a proper $U V^{n}$-surjection onto $M \times[0,1)$ and hence onto the product $L \times[0,1)$ as well. Thus, by Proposition 1.1, the inverse image $f^{-1}(L \times[0,1))$ and $\operatorname{Ker}(M)$ are homeomorphic. Consequently, to finish the proof it remains to construct an open embedding $h: f^{-1}(L \times[0,1)) \rightarrow N$ such that the complement $A=N-h\left(f^{-1}(L \times[0,1))\right.$ is a $Z$-set in $N$.

Now we shall follow the proof of Theorem 20.1 from [2]. First of all note that since $N$ is a Menger $(n+1)$-manifold, by Theorem 1.1, there is a countable subset $\left\{\varphi_{k}: k=1,2, \ldots\right\}$ of $C\left(I^{n+1}, N\right)$ consisting of $Z$-embeddings. As it was observe above, $f^{-1}(L \times\{0\})$ is a $Z$-set in $N$ and the inclusion $f^{-1}(L \times\{0\}) \hookrightarrow N$ is an $n$-homotopy equivalence. It easily follows from [3] that in this case there exists a retraction $r_{1}: N \rightarrow f^{-1}(L \times\{0\})$ that is $n$ homotopic to $\mathrm{id}_{N}$. Consider the restriction 


$$
s_{1}=r_{1} \mid \cdots: f^{-1}(L \times\{0\}) \cup \varphi_{1}\left(I^{n+1}\right) \rightarrow f^{-1}\left(L \times\left[0,2^{-1}\right)\right) .
$$

Clearly, $s_{1}$ is a proper map. By $1.1, s_{1}$ is properly $n$-homotopy to a $Z$ embedding

$$
g_{1}: f^{-1}(L \times\{0\}) \cup \varphi_{1}\left(I^{n+1}\right) \rightarrow f^{-1}\left(L \times\left[0,2^{-1}\right)\right)
$$

that coincides with the identity map on $f^{-1}(L \times\{0\})$. By Theorem 1.2 , there exists a homeomorphism $G_{1}: N \rightarrow N$ extending $g_{1}$. Put $h_{1}=G_{1}^{-1}$. Then $h_{1}$ is a homeomorphism such that

(a) $h_{1} \mid f^{-1}(L \times\{0\})=\mathrm{id}$,

(b) $\varphi_{1}\left(I^{n+1}\right) \subset h_{1} f^{-1}\left(L \times\left[0,2^{-1}\right)\right)$.

Consider now the polyhedron $K-\left(L \times\left[0,2^{-1}\right)\right)$. Since the set $L \times\left\{2^{-1}\right\}$ is a $Z$-set in $K-\left(L \times\left[0,2^{-1}\right)\right)$, we can conclude, by Theorem 1.6 , that the set $h_{1} f^{-1}\left(L \times\left\{2^{-1}\right\}\right)$ is a $Z$-set in a Menger $(n+1)$-manifold $N-$ $h_{1} f^{-1}\left(L \times\left[0,2^{-1}\right)\right)$. Moreover, since the inclusion $\left(L \times\left\{2^{-1}\right\}\right) \hookrightarrow K-$ $\left(L \times\left[0,2^{-1}\right)\right)$ is a homotopy equivalence, we conclude that the inclusion $h_{1} f^{-1}\left(L \times\left\{2^{-1}\right\}\right) \hookrightarrow N-h_{1} f^{-1}\left(L \times\left[0,2^{-1}\right)\right)$ is an $n$-homotopy equivalence. Again using the above construction, there is a homeomorphism $h_{2}^{\prime}$ : $N-h_{1} f^{-1}\left(L \times\left[0,2^{-1}\right)\right) \rightarrow N-h_{1} f^{-1}\left(L \times\left[0,2^{-1}\right)\right)$, which is the identity on $h_{1} f^{-1}\left(L \times\left\{2^{-1}\right\}\right)$ and for which

$$
\varphi_{2}\left(I^{n+1}\right) \cap\left(N-h_{1} f^{-1}\left(L \times\left[0,2^{-1}\right)\right)\right) \subset h_{2}^{\prime} h_{1} f^{-1}\left(L \times\left[2^{-1}, 2 \cdot 3^{-1}\right)\right) .
$$

Extend $h_{2}^{\prime}$ to a homeomorphism $h_{2}$ of all of $N$ by defining $h_{2}=$ id on $h_{1} f^{-1}\left(L \times\left[0,2^{-1}\right]\right)$. Then we have

$$
\varphi_{1}\left(I^{n+1}\right) \cup \varphi_{2}\left(I^{n+1}\right) \subset h_{2} h_{1} f^{-1}\left(L \times\left[0,2 \cdot 3^{-1}\right)\right) .
$$

Inductively continuing this process, we construct homeomorphism $h_{k}: N \rightarrow$ $N$ such that $h_{k+1}=\mathrm{id}$ on $h_{k} f^{-1}\left(L \times\left[0, k(k+1)^{-1}\right]\right)$ and

$$
\varphi_{1}\left(I^{n+1}\right) \cup \cdots \cup \varphi_{k}\left(I^{n+1}\right) \subset h_{k} h_{k-1} \cdots h_{1} f^{-1}\left(L \times\left[0, k(k+1)^{-1}\right)\right) .
$$

Define an open embedding $h: f^{-1}(L \times[0,1)) \rightarrow N$ by $h(x)=\lim _{k \rightarrow \infty} h_{k} \cdots$ $h_{1}(x)$ for each $x \in f^{-1}(L \times[0,1))$. Clearly

$$
\bigcup\left\{\varphi_{k}\left(I^{n+1}\right): k=1,2, \ldots\right\} \subset h \cdot f^{-1}(L \times[0,1))
$$

and consequently, by the choice of the family $\left\{\varphi_{k}: k=1,2, \ldots\right\}$, the complement $N-h \cdot f^{-1}(L \times[0,1))$ is a $Z$-set in $N$.

Now we can prove our main result, which is an analogue of Chapman's theorem on $[0,1)$-stable classification of Hilbert cube manifolds by homotopy type.

Theorem 2.2. Menger $(n+1)$-manifolds are $n$-homotopy equivalent if and only if their n-homotopy kernels are homeomorphic.

Proof. Let $M$ and $N$ be $n$-homotopy equivalent Menger $(n+1)$-manifolds. Fix maps $\alpha: M \rightarrow N$ and $\beta: N \rightarrow M$ such that $\beta \cdot \alpha \simeq^{n} \operatorname{id}_{M}$ and $\alpha \cdot \beta \simeq^{n} \mathrm{id}_{N}$. As above there are $(n+1)$-invertible proper $U V^{n}$-surjections $f: \operatorname{Ker}(M) \rightarrow$ $M \times[0,1)$ and $g: \operatorname{Ker}(N) \rightarrow N \times[0,1)$. Let $\varphi: M \times[0,1) \rightarrow N \times[0,1)$ and $\psi: N \times[0,1) \rightarrow M \times[0,1)$ be proper maps such that $\varphi$ is homotopic to $\alpha \times$ id 
and $\psi$ is homotopic to $\beta \times$ id, where id denotes the identity map of $[0,1)$ (see [2, Lemma 21.1]). Since $g$ is $(n+1)$-invertible and $\operatorname{dim} \operatorname{Ker}(M)=n+1$, there exists a proper map $r: \operatorname{Ker}(M) \rightarrow \operatorname{Ker}(N)$ such that $g \cdot r=\varphi \cdot f$. Similarly we fix a proper map $s: \operatorname{Ker}(N) \rightarrow \operatorname{Ker}(M)$ such that $f \cdot s=\psi \cdot g$. In this situation one can directly verify that $s \cdot r \simeq^{n} \operatorname{id}_{\operatorname{Ker}(M)}$ and $r \cdot s \simeq{ }^{n} \mathrm{id}_{\operatorname{Ker}(N)}$. Moreover, by Theorem 1.1, we can additionally suppose that $r$ and $s$ are $Z$-embeddings.

Now consider the $Z$-set $r(\operatorname{Ker}(M))$ in an Menger $(n+1)$-manifold $\operatorname{Ker}(N)$. It follows immediately from the above construction that the inclusion $r(\operatorname{Ker}(M))$ $\hookrightarrow \operatorname{Ker}(N)$ is an $n$-homotopy equivalence. By Proposition 2.4, there is a $Z$-set $A$ in $\operatorname{Ker}(N)$ such that $\operatorname{Ker}(N)-A$ is homeomorphic to $\operatorname{Ker}(r(\operatorname{Ker}(M)))=$ $\operatorname{Ker}(\operatorname{Ker}(M))$ (recall that $r$ is an embedding). Then, by Proposition 2.2 and 2.3 of $\S 2, \operatorname{Ker}(M) \approx \operatorname{Ker}(\operatorname{Ker}(\operatorname{Ker}(M))) \approx \operatorname{Ker}(\operatorname{Ker}(N)-A) \approx \operatorname{Ker}(N)$ as desired.

The second part of the theorem is trivial.

\section{REFERENCES}

1. M. Bestvina, Characterizing $k$-dimensional universal Menger compacta, Mem. Amer. Math. Soc., vol. 71, no. 380, Amer. Math. Soc., Providence, RI, 1988.

2. T. A. Chapman, Lectures on Hilbert cube manifolds, C.B.M.S. Regional Conf. Ser. in Math., vol. 28, Amer. Math. Soc., Providence, RI, 1976.

3. A. Chigogidze, Compacta lying in the n-dimensional universal Menger compactum and having homeomorphic complements in it, Mat. Sb. 133 (1987), 481-496; English transl., Math. USSR-Sb. 61 (1988), 471-484.

4. __ n-shapes and n-cohomotopy groups of compacta, Mat. Sb. 180 (1989), 322-335; English transl., Math. USSR-Sb. 66 (1990), 329-342.

5. __ Theory of $n$-shape, Uspekhi Mat. Nauk 44 (1989), 117-140; English transl., Russian Math. Surveys 44 (1989), 145-174.

6. __ n-soft maps of n-dimensional spaces, Mat. Z. 46 (1989), 88-95. (Russian)

7. _ $U V^{n}$-equivalence and n-equivalence (to appear).

8. A. N. Dranishnikov, Universal Menger compacta and universal maps, Mat. Sb. 129 (1986), 121-139; English transl., Math. USSR-Sb. 57 (1987), 131-150.

9. R. Engelking, Dimension theory, PWN, Warsaw, 1978.

10. R. H. Fox, On the Lusternik-Schnirelman category, Ann. of Math. (2) 42 (1941), 333-370.

11. R. C. Lacher, Cell-like mappings and their generalizations, Bull. Amer. Math. Soc. 83 (1977), 495-552.

12. J. H. C. Whitehead, Combinatorial homotopy. I, II, Bull. Amer. Math. Soc. 55 (1949), 213-245, 453-496.

Department of Mathematics, Moscow State University, Moscow, 119899

Current address: Department of Mathematics and Astronomy, University of Manitoba, Winnipeg, Manitoba, Canada R3T 2N2 